\section{Riesgo de enfermedades respiratorias en niños nacidos por cesárea}

El número de partos por cesárea continúa creciendo tanto en países desarrollados como en países en desarrollo y se han informado tasas de hasta 50\% en algunos países de América Latina. En los últimos años se ha observado un aumento en la proporción de partos por cesáreas electivas —es decir, sin una justificación obstétrica o clínica clara y generalmente aceptada-, a pesar de los riesgos que este tipo de parto implica ya sea para la madre o para el recién nacido.

En este trabajo se analiza la asociación entre las cesáreas electivas y la morbilidad respiratoria en los recién nacidos de una numerosa cohorte de mujeres embarazadas y se evalúa el efecto de la edad gestacional en el momento de la cesárea sobre la aparición de los trastornos respiratorios.

Para ello se estudiaron 34458 recién nacidos vivos, sin malformaciones congénitas, de partos sencillos de 37 a 41 semanas de gestación. Estos partos se registraron entre enero de 1998 y diciembre de 2006 y son parte de un estudio de cohorte más amplio realizado por el Hospital Universitario de Aarhus, Dinamarca, que mantuvo un estrecho seguimiento de 80000 embarazadas y recién nacidos a partir de septiembre de 1989 .

El riesgo de enfermedades respiratorias neonatales después de una cesárea electiva fue de dos a cuatro veces mayor que después de un nacimiento vaginal de igual edad gestacional de entre 37 y 39 semanas. Al realizar la comparación con el riesgo observado después de un nacimiento vaginal a las 40 semanas de gestación, el riesgo de enfermedades respiratorias fue siete veces mayor en los nacidos por cesárea electiva a las 37 semanas y tres veces mayor en los nacidos por esta vía a las 38 semanas. No se encontraron diferencias significativas entre los nacidos por cesáreas electivas a las 39 semanas y los nacidos por vía vaginal a las 40 semanas.

Esto puede deberse al efecto de las hormonas, ya que durante el trabajo de parto disminuye la producción de líquido pulmonar en el feto y aumenta su absorción, mientras crece la producción de sustancias surfactantes. Algunos autores han sugerido que la administración de corticosteroides a las embarazadas en la etapa previa a la cesárea podría reducir la aparición de enfermedades respira- torias en el recién nacido, sin embargo, esto no ha sido demostrado fehacientemente.

Estos resultados respaldan la hipótesis de que el riesgo de sufrir enfermedades respiratorias, entre ellas las de mayor gravedad, aumenta en los recién nacidos por cesárea electiva. Se encontró una reducción significativa en el riesgo de enfermedades respiratorias neonatales cuando la cesárea se realizó en la semana 39 de gestación. Sin embargo, la postergación de la cesárea puede elevar el número de cesáreas de emergencia en el momento del parto y el riesgo de algunas complicaciones, como la ruptura uterina en mujeres con una cesárea anterior, infecciones e incluso la muerte de la madre. El riego de enfermedades respiratorias aumentó en los recién nacidos por cesáreas de emergencia a medida que avanzaba la edad gestacional. Por el contrario, el trabajo de parto o la ruptura de las membranas antes de la cesárea pueden ser benéficos para el recién nacido.

A pesar de que se requieren estudios controlados aleatorizados en grandes poblaciones para establecer con mayor precisión el efecto de la edad de gestación y de las cesáreas practicadas durante el trabajo de parto sobre las enfermedades respiratorias en los recién nacidos, estos resultados indican que se puede reducir la morbilidad neonatal por enfermedades respiratorias si las cesáreas electivas se realizan en la semana 39 de gestación. Esta información es de gran importancia para las mujeres que planean tener un parto por cesárea electiva y debe ser parte del proceso de asesoría que brindan los profesionales de la salud a las embarazadas. Hansen AK, Wisborg K, Uldbjerg N, Henriksen TB. Risk of respiratory morbidity in term infants delivered by elective caesarean section: cohort study. Br Med J. 2008;336:85-7.)

\section{La infección por el VIH en Nicaragua}

La epidemia de infección por el virus de inmunodeficiencia humana (VIH) y el sida han devastado ya muchas familias y comunidades y constituye uno de los principales problemas de salud pública en el mundo. Según estimados del Programa Conjunto de las Naciones Unidas sobre el VIH/Sida y la Organización Mundial de la Salud, en el año 2004 había 39,4 millones de personas infectadas por el VIH, de ellas 1,7 millones vivían en América Latina y 400000 en el 
Caribe. A pesar de que en América Central esta epidemia no ha sido suficientemente estudiada, su efecto ha sido muy negativo y se espera que empeore. Cuatro de los seis países latinoamericanos de mayor prevalencia de infección por el VIH son centroamericanos: Belice $(2,0 \%)$, Honduras $(1,6 \%)$, Panamá $(1,5 \%)$ y Guatemala $(1,0 \%)$.

Nicaragua es el segundo país más pobre de América Latina y uno de los de mayor crecimiento demográfico de las Américas. Aunque la prevalencia estimada de la infección por el VIH en adultos era de $0,2 \%$ en 2001 , existen todas las condiciones para que la epidemia se recrudezca, entre ellas una elevada movilidad de la población y una cultura que estigmatiza el uso del condón.

En este artículo se describe la epidemia de $\mathrm{VIH} /$ sida en Nicaragua y se discuten las razones del incremento en el número de casos notificados en comparación con otros países de América Central. Para ello se tomaron en cuenta todos los casos detectados en el país entre 1987 (cuando se detectó el primero) y diciembre de 2004, según los datos del Programa Nacional de Enfermedades de Transmisión Sexual, VIH y sida.

Se observó un aumento constante en la incidencia de la infección por el VIH con una disminución entre 1993 y 1998. A partir de ese año, la incidencia aumentó abruptamente hasta llegar a ser 22 veces mayor que la observada entre 1987 y 1992. A pesar de las limitaciones del sistema de vigilancia, el número de casos ha aumentado, especialmente en las zonas urbanas y las regiones centroocidental y oriental del país, con un máximo de 54,2 por 100000 habitantes. Las provincias de Chinandega (350 000 habitantes), situada en la parte occidental de Nicaragua, y Managua (1 094000 habitantes) son las más afectadas.

La mayor parte de los casos (91\%) adquirieron la infección por la vía sexual (65\% eran heterosexuales y $26 \%$, homosexuales), mientras $4 \%$ se administraba drogas intravenosas y $3 \%$ se infectó perinatalmente.

La mayor tasa de infección se encontró en personas de 20-49 años de edad, especialmente en hombres de 20-44 años, con un pico entre 35 y 39 (incidencia de 125,6 por 100000 personas), y en mujeres de 20-34 años con un pico entre 20 y 24 (incidencia de 46,6 por 100000 personas). La relación de la infección entre hombres y mujeres fue de 3:1.

La distribución de casos de infección por el $\mathrm{VIH} /$ sida fue mayor en trabajadores de servicio, obreros, amas de casa y personas vinculadas con el comercio sexual $(32,8 \%, 17,4 \%, 16,4 \%$ y $6,0 \%$, respectivamente).

La mortalidad fue estable hasta 1999, pero aumentó desde entonces hasta 2004, cuando se introdujo en Nicaragua el tratamiento antirretroviral de gran actividad (HAART). Sin embargo, se observó cierto aumento en 2005.

La renuencia al uso del condón constituye un obstáculo para cualquier posible estrategia de prevención de la infección por el VIH.

La situación actual constituye un reto para las instituciones y la sociedad nicaragüenses. Se requiere cambiar las actitudes y prácticas de las poblaciones en riesgo para reducir la vulnerabilidad del país a la epidemia de VIH/sida. Es necesario mejorar el sistema nacional de vigilancia epidemiológica y realizar estudios de prevalencia que permitan disponer de estimados más reales del alcance de esta epidemia en el país. Para evitar la diseminación de esta infección es necesario realizar campañas educativas, especialmente en hombres y mujeres laboralmente activos. (Matute AJ, Delgado E, Amador JJ, Hoepelman AIM. The epidemiology of clinically apparent HIV infection in Nicaragua. Eur J Clin Microbiol Infect Dis. 2008;27:105-8.)

\section{La toxicidad por radioterapia y la calidad de vida en mujeres con cáncer ginecológico}

El cáncer ginecológico es una de las neoplasias malignas más frecuentes y una de sus variantes, el cáncer cervicouterino, es el segundo más frecuente en mujeres en todo el mundo, especialmente en países en desarrollo. Una vez avanzado el cáncer, su tratamiento requiere por lo general de la combinación de varios tipos de intervenciones, como la cirugía, la radioterapia y la quimioterapia. Todos estos procedimientos pueden tener efectos colaterales (estenosis vaginal, alteraciones en los intestinos, la vejiga y los ovarios, trastornos del sueño y síntomas de menopausia, entre otros) que pueden contribuir a que las pacientes tengan una peor percepción de su calidad de vida.

Se realizó un estudio prospectivo longitudinal en una cohorte de 107 mujeres de 18 a 75 años de edad con diagnóstico de cáncer cervicouterino o de endometrio para investigar la incidencia de la toxicidad aguda producida por la radioterapia en la calidad de vida percibida por las pacientes e identificar los factores predictivos asociados.

La toxicidad aguda se evaluó según los Criterios Generales de Toxicidad y las recomendaciones del Grupo de Radioterapia Oncológica, ambos del Instituto Nacional del Cáncer de los Estados Unidos de América. Para evaluar la calidad de vida se aplicó la encuesta abreviada de la Organización Mundial de la Salud antes y al terminar la radioterapia, así como durante el seguimiento clínico.

Según los resultados del análisis de regresión linear multifactorial para la salud general, hubo un efecto negativo significativo de la toxicidad de las 